\title{
Discussion
}

\section{Vascular disease, Parkinson's, and now Alzheimer's - Is homocysteine the new all-around bad guy?}

http://www.alzforum.org/res/for/journal/mattson/mattsan _transcript.asp

\section{Live Discussion held 30 April 2002}

Participants: Mark Mattson, Anthony White, Andrew McCaddon, Eugene Rogers, Tom Shea, Larry Sparks, Keith Crutcher, Gladys Maestre, Amy B. Graves, Paul Aisen, Monique Breteler, Ralph Green, Gabrielle Strobel

Gabrielle Strobel: Hello, and welcome everyone. I am Gabrielle Strobel, managing editor of the Alzforum, and will moderate today's discussion.

Mark Mattson: Mark Mattson, National Institute on Aging and Johns Hopkins University.

Amy B. Graves: I am Amy Borenstein Graves, Professor in Epidemiology and Biostatistics at the University of South Florida, doing work in risk factors for Alzheimer's disease (AD).

Eugene Rogers: I am from UMass Lowell.

Tom Shea: Biology, UMass Lowell. Gene Rogers and I have been studying homocysteine toxicity in culture and folate deprivation in culture and in apolipoprotein E (ApoE) knockout mice.

Andrew McCaddon: I am a general practitioner in North Wales with an interest in B12 deficiency and AD.

Keith Crutcher: I am at the University of Cincinnati.

Larry Sparks: I am from the Sun Health Research Institute, Sun City, Arizona. I am running the AD cholesterol-lowering treatment trial.

\footnotetext{
${ }^{1}$ Note: The transcript has been edited for clarity and accuracy.
}

Paul Aisen: I am from Georgetown University. I am conducting studies of homocysteine reduction in $\mathrm{AD}$.

Anthony White: I am at Imperial College, London, looking at interactions between homocysteine and metals, and their effects on neurons.

Gabrielle Strobel: Why not start by asking our guest of honor, Mark Mattson, to restate briefly what he thinks we know now about how homocysteine affects neurons in $\mathrm{AD}$ and other neurologic conditions.

Mark Mattson: Cell culture and in vivo data from mouse models of AD suggest that homocysteine can directly endanger/damage neurons. It enhances DNA damage by reducing repair and by promoting oxidative stress. The literature on the role of homocysteine in one-carbon metabolism is consistent with such a mechanism.

Gabrielle Strobel: Just to throw in a brand-new literature update: Stroke later this week will run a paper by Lydia Bazzano et al. [1] showing high dietary folate decreases stroke risk. The 9,764 participants in the NHEFS nutrition survey were asked about eating habits five times over 10 years. No surprise there.

Mark, the homocysteine-A $\beta$-DNA damage link is new and intriguing. How could that work? Do you know which repair processes homocysteine interferes 
with? Does it inhibit any of the known repair DNA enzymes?

Mark Mattson: In our hands, homocysteine increases both uracil misincorporation and oxidative DNA base modifications. We are currently determining whether UDGs or FPGs, Ku proteins, etc., are affected by homocysteine. We do know that PARP and p53 are players in the cell death cascade induced by homocysteine.

Gabrielle Strobel: Mark, Ku proteins - that is interesting. Do postmitotic neurons have double-strand breaks?

Mark Mattson: Yes, they can have double-strand breaks. Also, $\mathrm{Ku}$ localizes to telomere ends, and we are finding that (in embryonic neurons) telomerase is a "guardian of the genome" that protects against DNA damage.

Gabrielle Strobel: What happens with a neuron that has double-strand breaks and tries to reenter the cell cycle, as some are finding (see [2]). Can it repair these breaks?

Mark Mattson: Gabrielle, the cell cycle reentry hypothesis is alive and well. Since neurons are already postmitotic, the attempt to reenter the cell cycle may trigger apoptosis.

Amy B. Graves: To what extent do people feel the homocysteine/folate link with $\mathrm{AD}$ is responsible for the cardiovascular association with AD?

Andrew McCaddon: Amy, I think the elevated homocysteine reflects a pathogenic mechanism common to both - namely, the effects of oxidative stress on B12 metabolism.

Larry Sparks: Our work has shown that most AD subjects have heart disease and that many individuals with heart disease have AD-like neuropathology. It might be hard to separate AD-heart disease and homocysteine in that vein.

Amy B. Graves: So, Andrew, you think the homocysteine link is working independently of the rest of the cardiovascular piece?

Gladys Maestre: Our work in a population sample studied extensively from the cardiovascular disease suggests that the risk for AD is independent of the cardiovascular effect.

Amy B. Graves: Gladys, can you clarify what you mean with cardiovascular disease and the cardiovascular effect?

Gladys Maestre: The risk of AD conferred by homocysteine is independent. It is still strong even when we account for hypertension, cardiovascular events, history of stroke, lipids, and even blood pressure, circadian dysregulation, arrhythmias.

Amy B. Graves: Gladys and Paul, this is important. Maybe it implies different causative mechanisms.

Paul Aisen: Amy, it is consistent with a direct contribution of homocysteine to amyloid neurotoxicity.

Amy B. Graves: Paul, so that means that homocysteine is directly involved in amyloid deposition?

Paul Aisen: No, I think it is more likely that homocysteine increases the sensitivity of neurons to amyloidmediated damage.

Eugene Rogers: In cell culture, neurotoxicity occurs with high homocysteine independently, of course, because there are no vascular issues.

Andrew McCaddon: Mark, we have a paper in the next edition of Neurology presenting a hypothesis complementary to yours - namely, that oxidative stress might contribute to elevated serum homocysteine ([3], see also [4]).

Mark Mattson: Andrew, your findings make sense and would fit with Tom Shea's studies in ApoE knockout mice, which seem to have decreased antioxidant capacity.

Tom Shea: If we co-treated with S-adenosyl methionine, homocysteine-induced apoptosis was prevented depletion of essential methylation of DNA due to homocysteine accumulation?

Mark Mattson: One interesting question is: Under conditions of folate deficiency, do neurons themselves generate the homocysteine that may eventually kill them? Does folate deficiency have different effects on homocysteine production in different cell types? 
Eugene Rogers: Folate deficiency has different effects on different cell types, e.g., transulfuration and betaine-homocysteine methyl transferase pathways are essentially absent in neurons, so homocysteine removal is basically dependant on remethylation to methionine with methylene tetrahydrofolate reductase and methionine synthase, or on cellular export, where it may accumulate in the extracellular fluid or the CNS.

Anthony White: We have also found that increases in oxidative stress or a reduction in antioxidant levels in cultured neurons makes them highly vulnerable to homocysteine. This would fit with Andrew's theory.

Mark Mattson: Alterations in lipid (shingolipids and cholesterol) metabolism and oxidative stress are common to many different age-related diseases, including cardiovascular disease and AD. My take on this is that these alterations might differentially occur in blood vessels versus neurons, but more often occur simultaneously.

Paul Aisen: The paper from the Oxford group published a few years back in Archives of Neurology showed a strong link between homocysteine levels and neuropathologically pure $\mathrm{AD}$ [5]. That is, the homocysteine-AD link seems to be independent of vascular disease.

Andrew McCaddon: We also now have some interesting data accepted showing elevated serum cysteine in our AD patients, and a correlation between cognitive scores and total serum glutathione [3]. Our interpretation was that we are seeing the effects of oxidative stress on methionine synthase and cystathionine $\beta$-synthase activity.

Larry Sparks: Was there a negative relationship between glutathione levels and cognitive scores, and what instruments were used?

Anthony White: Andrew, loss of glutathione in our neurons increases toxicity of homocysteine and cysteine (the latter to a lesser extent).

Eugene Rogers: Anthony, do you first observe an initial increase in neuronal glutathione before depletion and then enhanced toxicity to homocysteine and cysteine?

Anthony White: Gene, we have not tested the full time course, but subtoxic levels of homocysteine do not appear to alter glutathione levels in our neurons early on.

Andrew McCaddon: All, our "functional" vitamin B12 deficiency hypothesis requires in vitro testing.

Mark Mattson: Andrew, we could address the B12 issue in our hippocampal cultures.

Andrew McCaddon: Yes! Excellent, Mark. What you need to do is culture those neurons in a homocysteine-free medium, and then "oxidatively stress" them - they should pump the stuff out into the medium.

Tom Shea: Homocysteine does synergistically enhance $\mathrm{A} \beta$ generated ROS and apoptosis [6].

Gladys Maestre: Homocysteine really increments with age and is higher in men, but there are no real data on why this might be - any thoughts?

Mark Mattson: Since oxidative stress increases with age, one could propose that the increased homocysteine levels are secondary to oxidative stress. Folate levels also tend to decrease with age, so there could be a diet/absorption/conversion problem during aging.

Tom Shea: We saw a large increase in homocysteine in culture medium following two-hour folate deprivation.

Mark Mattson: That is a very rapid increase in homocysteine. Makes you wonder what might be happening in the brain under various conditions of changing diet, stress, or injury.

Paul Aisen: Gladys, perhaps this is related to renal function.

Gladys Maestre: Paul, we explored that with creatinine levels and did not find any differences between genders.

Paul Aisen: But serum creatinine may not be an adequate indicator of glomerular filtration rate.

Eugene Rogers: Cystatin C will soon replace creatinine as a better indicator of renal clearance.

Andrew McCaddon: Gladys, I expect one reason it increases with age is because of the effects of oxidative stress on intracellular B12 metabolism. 
Gladys Maestre: Andrew, exactly what do you mean?

Andrew McCaddon: Anyone here heard of glutathionylcobalamin? This is the obligate intracellular intermediate of B12 metabolism and requires reduced glutathione for its synthesis. So, not only will oxidative stress impair the methionine synthase reaction, it will prevent the intracellular reduction of the vitamin to its active state, too.

Tom Shea: Definitely. We did see increased glutathione in both normal and ApoE knockout CNS following one month of folate deprivation, but only the ApoE knockout showed increased thiobarbituric acid reagent substances (TBARS, markers of oxidative damage). Normal mice seem to be able to handle the problem. Makes me think so much is masked in normal aging.

Larry Sparks: Are homocysteine levels increased in any of the transgenic mouse models of AD?

Mark Mattson: Larry, we did not observe an increase in blood homocysteine levels in the line of $\mathrm{A} \beta \mathrm{PP}$ mutant mice we have been studying (Dave Borchelt's; Swedish mutation). We have not looked at brain levels, yet.

Tom Shea: We did see increased blood homocysteine in our mice fed a folate-deprived diet.

Larry Sparks: Has anyone ever shown accumulation of homocysteine in senile plaques?

Mark Mattson: Larry, also, the increase in blood homocysteine levels in $\mathrm{A} \beta \mathrm{PP}$ mutant mice on a folatedeficient diet was of a similar magnitude to that of nontransgenic mice on the folate-deficient diet.

Larry Sparks: Mark, any differences in cognitive ability or lack thereof induced?

Mark Mattson: Larry, yes. A folate-deficient diet exacerbates the spatial learning deficits in the $\mathrm{A} \beta \mathrm{PP}$ mutant mice.

Larry Sparks: Mark, did the folate diet produce the same effect in the nontransgenic mice, but to a lesser extent?

Gladys Maestre: Mark, could you reverse those learning deficits with folate?
Mark Mattson: Gladys, we have not yet tried to reverse the learning deficits.

Andrew McCaddon: Regarding A $\beta \mathrm{PP}$ - my whole interest in this started when we found low B12 levels in the $717 \mathrm{~A} \beta \mathrm{PP}$ mutation family. I gather this mutation is also associated with low intracellular glutathione, so this fits in nicely.

Gabrielle Strobel: Dave Holtzman has PDAPP $\mathrm{x}$ ApoE knockout mice that express individual human ApoE alleles. Would it make sense to test the E2, E3, and E4 mice in your folate diet/iron challenge paradigm to get at in vivo mechanisms of the human genetic ApoE4 risk?

Tom Shea: Definitely.

Gabrielle Strobel: Mark, would such mice be interesting in your experiment with DNA repair and $\mathrm{A} \beta$ toxicity?

Mark Mattson: Gabrielle, studies of DNA repair and $\mathrm{A} \beta$ toxicity in the $\mathrm{A} \beta \mathrm{PP}$-mutant mice are under way. We eventually plan to cross the A $\beta$ PP-mutant mice with mice that have altered DNA repair (knockouts and transgenics for specific DNA repair proteins).

Anthony White: Mark, did you look at the effect of folate deficiency in $\mathrm{A} \beta \mathrm{PP}$-mutant mice in old age of your mice (about 18 months), but before amyloid deposition? Just wondering about aging effects in these mice.

Mark Mattson: Anthony, we only observed hippocampal neuron loss in mice on a folate-deficient diet in $\mathrm{A} \beta \mathrm{PP}$-mutant mice in which amyloid deposition was beginning (8-10 months) or extensive (15 months).

Anthony White: Mark, was the cell loss associated with amyloid deposits, or could it be related to aggregated but not "deposited" amyloid?

Mark Mattson: Anthony, the cell loss first occurs at a time when $\mathrm{A} \beta$ is beginning to be deposited, but prior to the presence of overt plaque-like deposits. I believe the peptide is most toxic during the process of aggregation, and once deposited, its toxicity decreases. We really do think the peptide generates radicals, by a mechanism that requires iron or copper ions. 
Anthony White: Mark, I agree. Was there any damage elsewhere in the brain or just hippocampus? Perhaps more sensitive measure of cell dysfunction should be examined.

Mark Mattson: Anthony, we have no data on other brain regions. The analyses have to be done using stereological counting methods, which are more difficult to apply to (e.g.,) cortex.

Paul Aisen: Mark, based on your work, do you think homocysteine reduction would slow progression of $\mathrm{AD}$ ?

Andrew McCaddon: Have not done a formal clinical trial yet, but my personal opinion after looking at this for the last 10 years now is that correcting the hyperhomocysteinemia in our patients by folate and B12 supplementation does not reverse cognitive decline, though it might slow the process slightly.

Andrew McCaddon: One curious thing regarding the folate link: Although serum folate is low, red cell folate is not low in our studies, and none of the patients have any evidence of anemia nor does the cognitive impairment precede anemia.

Paul Aisen: Andrew, I think it is clear that within normal ranges of folate, there is a negative association between folate level and homocysteine. So if homocysteine is neurotoxic, it would not be surprising to see cognitive effects without hematologic effects.

Andrew McCaddon: True, Paul, but why is red cell folate normal? I wonder if the low folate is secondary to a functional B12 deficiency, resulting in a failure to polyglutamate intracellular folate, and a consequent loss of folate in urine.

Paul Aisen: Andrew, I suspect that the simplest explanation - that the main determinant of folate levels is dietary - is most likely. We are just beginning to look at this in an AD population.

Eugene Rogers: Andrew, serum folate may reflect more acute exposure to dietary folate as opposed to red cell levels, which are more of a long-term - and perhaps better - indicator of functional folate deficiency.

Monique Breteler: I do not know about deficiencies, but we clearly did not see a relation with dietary intake.
Gabrielle Strobel: Monique, is your comment in response to my earlier question about ApoE genotypes?

Monique Breteler: No, about folate intake. I do not know about ApoE genotype at this moment.

Gladys Maestre: Monique, we also did not see a relation with dietary intake, even though we went to every home to obtain dietary information.

Gabrielle Strobel: Gladys and Monique, what do you mean by no relation to dietary intake? No matter how much folate you eat with your foods, your body regulates homocysteine levels independent of diet? I do not understand.

Monique Breteler: Neither do I. Maybe the dietary intake data do not properly reflect what you get as far as folate is concerned? We do not have blood levels.

Amy B. Graves: Monique and Gabrielle, this is not so unusual. We see no relation between serum cholesterol and dietary intake from a food frequency survey in two studies in Florida, one in Caucasians and the other in African Americans.

Gladys Maestre: Gabrielle, it is hard to really know anyone's dietary intake, but we look at this issue very carefully and still, folate and vitamin B12 levels do not correlate very well with intake. If you give a supplement, it is somehow different.

Andrew McCaddon: Just to throw some other things into the dietary discussion, we found that body mass index did not differ between our AD patient and control group. Not a wonderful nutritional status marker I know, but the best we could come up with.

Amy B. Graves: Andrew, body mass index (BMI) in prevalent cases may not be useful.

Mark Mattson: Andrew, based on our animal models and Richard Mayeux's data, we think high calorie intake will increase risk of AD. However, BMI is not a direct (or even good) measure of calorie intake.

Andrew McCaddon: Regarding BMI, I know. It was the best we could do in the circumstances, though we did measure retinol-binding protein in the earlier study and found no difference. 
Andrew McCaddon: Paul, fair enough, but what do you then make of the Nun Study findings?

Paul Aisen: Andrew, do you mean the predictive value of folate?

Andrew McCaddon: Paul, I mean, in the Nun Study, all the participants had exactly the same dietary intake of folate.

Mark Mattson: Andrew, we observe a consistent, modest, but significant decrease in homocysteine levels in rats or mice maintained on a dietary restriction regimen.

Paul Aisen: Andrew, diet may be the most important determinant of folate levels, but need not be the only one.

Andrew McCaddon: Mark, could the reduction in homocysteine levels in animals on dietary restriction be explained by a reduction in oxidative stress? It has taken me some time to realize this, but I think the bottom line is that homocysteine is, in fact, an excellent marker of oxidative stress in neuronal and vascular tissue.

Tom Shea: That is an interesting thought, Andrew.

Andrew McCaddon: Thanks, Tom. It does take a bit of a different stance on the whole thing.

Gabrielle Strobel: Andrew, is homocysteine specific to $\mathrm{AD}$ in any way, then, or does it apply equally to all diseases of aging?

Andrew McCaddon: Gabrielle, aging in general, though oxidative stress is increased in diseases like AD.

Anthony White: Mark, we find that copper (even background levels) dramatically increases neuronal damage in the presence of homocysteine in cortical cultures. Have you looked at this in hippocampal cultures? Could the amyloid be supplying a source of transition metal for homocysteine reduction leading to toxicity?

Mark Mattson: Anthony, we have not looked at this, but the experiment would be to see if a copper chelator is protective.

Monique Breteler: Anthony, how and where could you measure copper in humans?
Anthony White: Monique, we have routinely been measuring copper in animal tissues including brain, liver, and serum using inductively coupled plasma mass spectrometry. This can easily be done on human samples.

Tom Shea: Anthony, any interaction with good old aluminum?

Anthony White: Tom, alas, we have not looked at that. Probably should, but it is a word that people do not always want to hear.

Tom Shea: I know!

Gabrielle Strobel: We agree there is enough research data to increase daily folate intake recommendations. To what levels, and do they vary for cardiovascular and dementia prevention? Does this even make sense in the absence of a proven relationship between dietary intake and serum levels?

Paul Aisen: Gabrielle, I would argue that we need clinical trials.

Gabrielle Strobel: Is eating large amounts of folate safe? No such thing as overdosing on spinach pie, perhaps, but how about people bingeing on folate supplements?

Paul Aisen: Gabrielle, large amounts of folate can precipitate manifestations of B12 deficiency.

Monique Breteler: I disagree that we have evidence that increasing folate will help prevent dementia.

Gabrielle Strobel: Monique, do you think the epidemiology is insufficient? How about the recent Framingham study?

Monique Breteler: Very interesting observation, Framingham. But yes, we have no data suggesting that higher folate intake reduces risk.

Eugene Rogers: Paul, Andrew, and others: In relation to the Nun Study, CSF folate is concentrated from blood across the choroid plexus about fourfold, and it appears that serum or red cell folate are not predictors of CSF folate, and CSF may be associated with development of AD. Do you think that pathology of this region could play a role? 
Paul Aisen: Eugene, nonetheless, peripheral folate levels are related to risk of $\mathrm{AD}$.

Andrew McCaddon: Paul, yes, I think folate does play a role, but as I say, I wonder if the whole mechanism we are seeing is simply the effects of oxidative stress on the methionine synthase reaction, and on the intracellular processing of B12.

Larry Sparks: Andrew, do you think that one might find homocysteine in the vasculature of an individual with multiple sclerosis, then? I am asking because freeradical activity/oxidative stress in the vasculature can lead to inflammation, as we have shown for AD and in the brains of cholesterol-fed rabbits, and multiple sclerosis is a vascular inflammation disorder.

Andrew McCaddon: I have not looked at multiple sclerosis personally, but probably the answer would be yes.

Gabrielle Strobel: Larry, are you measuring folate levels in your trial?

Larry Sparks: No, but many of the apolipoproteins and $\mathrm{A} \beta$.

Gabrielle Strobel: Monique, what sort of trial do you want to see to find out if increased folate protects?

Monique Breteler: Prevention trials - not in MCI or demented people, but in nondemented.

Mark Mattson: I agree that prevention trials are critical, as trials in symptomatic patients are likely to have only a weak beneficial effect.

Gabrielle Strobel: Who would fund the Rolls Royce of trials for something as cheap as folate?

Gladys Maestre: Gabrielle, I think it is very important for clinicians to realize that it is worthwhile assessing homocysteine in a healthy subject and to take the opportunity to make a nutritional assessment. One of the problems is that, indeed, there are not clear-cut "normal levels" for any of these three elements, by age stratum and gender. So what to treat is not clear.

Paul Aisen: Gabrielle and others, prevention trials are attractive in theory, but vastly more expensive to conduct.
Gabrielle Strobel: Is prevention the key with folic acid supplementation? I am asking because initial treatment trials for NSAIDs and estrogen have failed, perhaps because the patients were too advanced in their disease. Do you think this would not happen with folate?

Paul Aisen: Gabrielle, but a trial of vitamin E did not fail [7].

Larry Sparks: If you want to do a prevention trial based on increasing folate in the appropriate compartment (where it will do the most good) you must have a consistent means to increase that pool. Also, I believe that the direction with prevention trials will be that one will have to show first that the agent to be tested is effective as a treatment, at least in mild to moderate AD.

Tom Shea: It was only in culture, but when we increased folate to five times the normal culture level, we blocked $\mathrm{A} \beta$-induced oxidative stress.

Anthony White: Tom, did that alter homocysteine levels in the cells/medium?

Tom Shea: Did not check, Anthony. Should have, will do>

Andrew McCaddon: If anyone wants to test the hypothesis, it would require culturing neurons in homocysteine-free medium, oxidatively stressing them, and measuring whether they export homocysteine into the medium. If anyone here can do that, I would be grateful to know what they find.

Tom Shea: Actually, Anthony, trouble there: We have undetectable levels of homocysteine (by HPLC) unless we deprive folate for a few hours, so may not be able to determine. Any ideas?

Anthony White: Tom, there may be other means of measurement, but not sure at present.

Andrew McCaddon: I saw a reference of a new assay that could detect nanomoles of reduced, free oxidized, protein-bound, and total homocysteine in human plasma [8].

Tom Shea: I will check that out.

Paul Aisen: Andrew, any thoughts on reduced folic acid as a supplement? 
Anthony White: Tom, have you looked at the redox status of the cultures treated with high folate levels? This may be related to Andrew's theory.

Tom Shea: Anthony, no, another thing that should be done.

Andrew McCaddon: There is another implication of our idea - namely, that the currently available forms of B12 might not be the best to use in neuropathologic or vascular disease. Glutathionylcobalamin might be preferable. Have you had a chance to do any more work on glutathionylcobalamin, Ralph?

Andrew McCaddon: I am afraid glutathionylcobalamin is not yet commercially available.

Ralph Green: Don Jacobsen is knowledgeable about glutathionylcobalamin.

Andrew McCaddon: I have had some good results with patients given B12/folate/and N-acetyl-cysteine (NAC), but only case studies at present.

Eugene Rogers: Interestingly, NAC has been used clinically for years (Mucomist) to treat acetaminophen overdose, which exerts toxicity via depletion of cellular glutathione.

Andrew McCaddon: Yes, that was our rationale for using NAC. We wanted to increase glutathione and B12 in our patients.

\section{References}

[1] L.A. Bazzano, J. He, L.G. Ogden, C. Loria, S. Vupputuri, L. Myers and P.K. Whelton, Dietary intake of folate and risk of stroke in US men and women: NHANES I Epidemiologic Follow-up Study. National Health and Nutrition Examination Survey, Stroke 33 (2002), 1183-1188.

[2] Alzheimer Research Forum, Transcript of Live Discussion: The Cell Cycle in Alzheimer's Disease: Let's Unite Around Division!, http://www.alzforum.org/res/for/journal/vincent/default.asp, 20 May 2002.

[3] A. McCaddon, B. Regland, P. Hudson and G. Davies, Functional vitamin B(12) deficiency and Alzheimer disease, Neurology 58 (2002), 1395-1399.

[4] A. McCaddon, The "cobalaminergic" hypothesis. Alzheimer Research Forum, http://www.alzforum.org/res/adh/cur/coblamin/coblaminergic.asp, October 2000.

[5] R. Clarke, A.D. Smith, K.A. Jobst, H. Refsum, L. Sutton and P.M. Ueland, Folate, vitamin B12, and serum total homocysteine levels in confirmed Alzheimer disease, Arch Neurol $\mathbf{5 5}$ (1998), 1449-1455.

[6] P.I. Ho, D. Ortiz, E. Rogers and T.B. Shea, Multiple aspects of homocysteine neurotoxicity: glutamate excitotoxicity, kinase hyperactivation and DNA damage, J Neurosci Res 70 (2002), 694-702.

[7] M. Sano, C. Ernesto, R.G. Thomas, M.R. Klauber, K. Schafer, M. Grundman, P. Woodbury, J. Growdon, C.W. Cotman, E. Pfeiffer, L.S. Schneider and L.J. Thal, A controlled trial of selegiline, alpha-tocopherol, or both as treatment for Alzheimer's disease. The Alzheimer's Disease Cooperative Study, N Engl J Med 336 (1997), 1216-1222.

[8] G. Chwatko and E. Bald, Determination of different species of homocysteine in human plasma by high-performance liquid chromatography with ultraviolet detection, J Chromatogr A 949 (2002), 141-151. 